Geometry $\&$ Topology

Volume 8 (2004) 1227-1242

Published: 22 September 2004

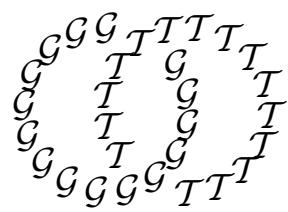

\title{
Tetra and Didi, the cosmic spectral twins
}

\author{
Peter G Doyle \\ JuAN PABlo Rossetti \\ Department of Mathematics, Dartmouth College \\ Hanover, NH 03755-3551, USA \\ and \\ FaMAF - Ciem, Universidad Nacional de Córdoba, Argentina \\ Email: doyle@math.dartmouth.edu and rossetti@mate.uncor.edu
}

\begin{abstract}
We introduce a pair of isospectral but non-isometric compact flat 3-manifolds called Tetra (a tetracosm) and Didi (a didicosm). The closed geodesics of Tetra and Didi are very different. Where Tetra has two quarter-twisting geodesics of the shortest length, Didi has four half-twisting geodesics. Nevertheless, these spaces are isospectral. This isospectrality can be proven directly by matching eigenfunctions having the same eigenvalue. However, the real interest of this pair - and what led us to discover it - is the way isospectrality emerges from the Selberg trace formula, as the result of a delicate interplay between the lengths and twists of closed geodesics.
\end{abstract}

AMS Classification numbers Primary: 57M50, 58J53

Secondary: $11 \mathrm{~F} 72,53 \mathrm{C} 22$

Keywords: Flat structure, 3-manifold, platycosm, Laplace spectrum, isospectral, Selberg trace formula, closed geodesic

Proposed: Walter Neumann

Seconded: Benson Farb, Steven Ferry
Received: 17 July 2003

Revised: 20 September 2004 


\section{Introducing Tetra and Didi}

A platycosm is a compact flat 3-manifold. Simplest among platycosms are the torocosms (the artifacts formerly known as ' 3 -dimensional tori'). Torocosms come in various shapes and sizes. Among these, we distinguish the cubical torocosm $\mathbf{R}^{3} / \mathbf{Z}^{3}$, and the two-story torocosm TwoTall $=\mathbf{R}^{3} /(\mathbf{Z} \times \mathbf{Z} \times 2 \mathbf{Z})$.

All other platycosms arise as quotients of torocosms. There are 10 distinct types in all, of which 6 (torocosm; dicosm; tricosm; tetracosm; hexacosm; didicosm) are orientable. The spaces themselves are well known, but the naming scheme, due to Conway, is new. The naming scheme and the spaces themselves are described in great detail by Conway and Rossetti in [2. The spaces are described under different names by Weeks [15], and Weeks (see [16]) has also produced software which allows you to 'fly around' inside these spaces, and many others as well.

Here we are concerned with two specific platycosms: Tetra, a tetracosm, and Didi, a didicosm. Please note that the prefix 'didi-' is a doubling of the prefix 'di-', and not some exotic Greek root. The word 'didicosm' is pronounced 'diedie-cosm', but Didi is pronounced 'Dee-dee'. Tetra and Didi turn out to be, up to scale, the unique pair of cosmic spectral twins (non-isometric platycosms with identical Laplace spectrum).

Tetra and Didi are both 4 -fold quotients of TwoTall. Tetra is the quotient of TwoTall by a fixed-point-free action of $\mathbf{Z} / 4 \mathbf{Z}$, while Didi is the quotient by a fixed-point-free action of $\mathbf{Z} / 2 \mathbf{Z} \times \mathbf{Z} / 2 \mathbf{Z}$. To get Tetra, we adjoin to the translation group $\mathbf{Z} \times \mathbf{Z} \times 2 \mathbf{Z}$ in $(x, y, z)$-space the quarter-turn screw motion

$$
\tau:(x, y, z) \mapsto(-y, x, z+1 / 2) .
$$

To get Didi, we adjoin instead the two half-turn screw motions

$$
\rho_{x}:(x, y, z) \mapsto(x+1 / 2,-y,-z)
$$

and

$$
\rho_{y}:(x, y, z) \mapsto(-x, y+1 / 2,1-z),
$$

which together with the translations generate a third half-turn screw motion

$$
\rho_{z}:(x, y, z) \mapsto(1 / 2-x, 1 / 2-y, z+1) .
$$

Both Tetra and Didi have as a fundamental domain the box

$$
[-1 / 2,1 / 2] \times[-1 / 2,1 / 2] \times[0,1 / 2],
$$

and in both cases the four vertical sides are glued up in parallel in the usual way, front to back and left to right, yielding a stack of square tori. The difference 

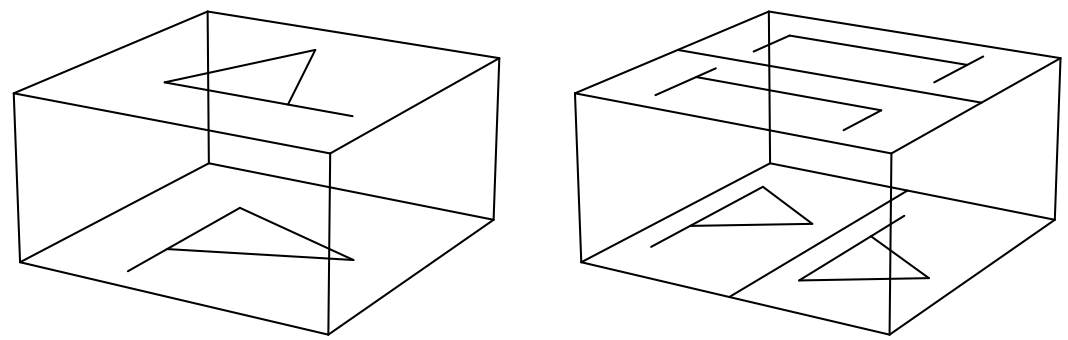

Figure 1: Tetra and Didi. The sides of the box glue back to front and left to right in the usual way; the tops and bottoms glue as indicated. Note that in the case of Didi, the top and bottom glue not to each other but each to itself, yielding two Klein bottles embedded in the quotient (which is nonetheless orientable!).

comes in the glueings of the top and bottom. (See Figure 1.) To get Tetra, you use $\tau$ to glue the bottom to the top with a quarter-turn. To get Didi, you use $\rho_{x}$ to glue the bottom to itself via a glide reflection, and $\rho_{y}$ to glue the top to itself via a glide reflection. These glueings produce two Klein bottles embedded in Didi. There is also a third, 'vertical' Klein bottle, associated to $\rho_{z}$.

Note We have described Tetra and Didi as quotients of a common 4-fold cover. In fact they have a common 2 -fold cover, the 'cubical dicosm', and a common 2-fold orbifold quotient. While both of these related spaces have a role to play in explaining the relationship between Tetra and Didi, we won't have any further occasion to discuss them here.

\section{Non-isometric}

Tetra and Didi are not isometric. In fact, since they have different fundamental groups, they are not even homeomorphic. (Tetra has first Betti number 1, while Didi has first Betti number 0.)

Moreover, in contrast to many of the known examples of spectral twins, their closed geodesics are markedly different. In a platycosm, when you go around a closed geodesic you come back twisted through some angle $\theta$. In a torocosm, this twist angle is always 0 . But in Tetra, the shortest geodesics have twist $\theta=\pi / 2$ : We call such geodesics quarter-twisting geodesics, or quarter-twisters. In Didi, the shortest geodesics have twist $\theta=\pi$ : We call such geodesics halftwisting geodesics, or half-twisters. (See Figure 2.) 
The fact that the shortest geodesics in Tetra are quarter-twisters while those in Didi are half-twisters already shows that these spaces are non-isometric. (Indeed, Didi has no quarter-twisting geodesics at all; this is an aspect of the fact that Tetra and Didi have different 'holonomy groups', namely $\mathbf{Z} / 4 \mathbf{Z}$ versus $\mathbf{Z} / 2 \mathbf{Z} \times \mathbf{Z} / 2 \mathbf{Z}$.)

Let us count the short geodesics in Tetra and Didi.

Warning When counting geodesics, we count each pair of oppositely-oriented geodesics only once.

In Tetra there are two quarter-twisting geodesics of length $1 / 2$, one running up the middle of the box along the line $x=y=0$, and one running up the four identified edges of the box. The vertical midlines of the four sides of the box combine to give a third geodesic, but this one is a half-twisting geodesic of length 1 .

In Didi, there are four half-twisting geodesics of length $1 / 2$, two associated with $\rho_{x}$ sitting in the Klein bottle gotten by glueing the bottom of the box, and two associated with $\rho_{y}$ sitting in the Klein bottle gotten by glueing the top. (See Figure 2]) In addition, there are two half-twisting geodesics sitting in the $\rho_{z}$ Klein bottle, but these have length 1.
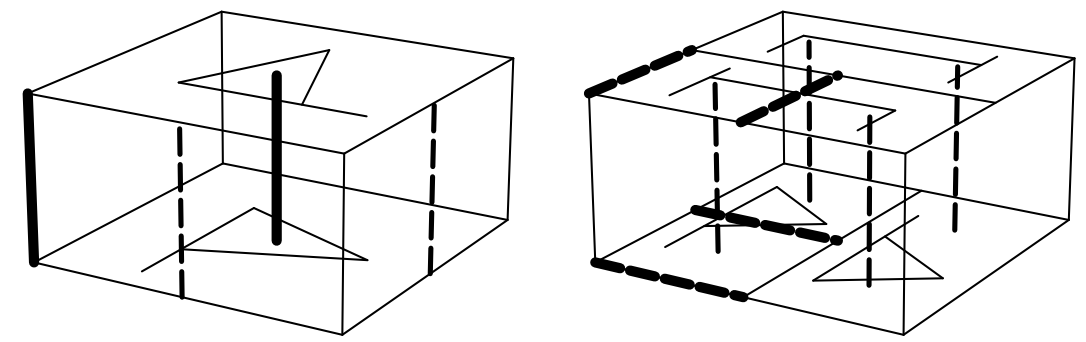

Figure 2: Twisted geodesics in Tetra and Didi. Solid thick segments close up into quarter-twisting geodesics of length $1 / 2$; dashed thick segments close up into halftwisting geodesics of length $1 / 2$; dashed thin segments join together in pairs to form half-twisting geodesics of length 1 . Note that in the case of Didi, if you start up one of the dashed thin vertical segments, you continue down the segment 'kitty-corner' to it.

Let's call a geodesic twisted if it has a nontrivial twist. We've located 3 twisted geodesics in Tetra, and 6 in Didi. These are all primitive, which means that they don't arise by going more than once around a shorter geodesic. In fact these are the only primitive twisted geodesics in Tetra and Didi. Of course there are also imprimitive twisted geodesics, which come from going around a 
half-twister an odd number of times, or around a quarter-twister a number of times not divisible by 4 .

Note To verify that we have identified all the twisted geodesics, observe that any twisted geodesic in Tetra (or Didi) unwraps to a straight line in $R^{3}$. Translating-with-a-twist along this straight line will be a 'covering transformation' - that is, one of the symmetries of $\mathbf{R}^{3}$ consistent with all patterns obtained by unwrapping patterns in the quotient Tetra (or Didi). The translation is by the length of the geodesic, and the twist is equal (and opposite) to the twist of the geodesic. In the case of Tetra, the line must be vertical, because all covering translations-with-a-nontrivial-twist run vertically. In the case of Didi, the line can run in any of the three coordinate directions. Look carefully at the possibilities, and you'll see that in listing twisted geodesics we've accounted for all of them.

\section{Isospectral}

While Tetra and Didi are not isometric, they are isospectral. By definition, two spaces are isospectral if there exists some way of matching up the eigenfunctions of the Laplacian of the two spaces so that corresponding eigenfunctions have the same eigenvalue. In this section, we will show that Tetra and Didi are isospectral by describing such a correspondence.

We are giving this explicit proof because it is entirely elementary - it relies only on linear algebra and Fourier series - and because it is illuminating in its own way. Other, more 'conceptual' proofs are available. Further along, we will outline one such proof, by way of the Selberg trace formula. A third proof can be obtained using the general machinery for flat manifolds developed by Miatello and Rossetti in [8], and a fourth using the 'dual' approach of [9]. A close relative of this fourth proof emerges naturally in the proof that Tetra and Didi are the unique pair of cosmic spectral twins [12, discussed briefly below.

A function on Tetra corresponds to a function $f$ on TwoTall that is invariant under $\tau$, in that

$$
f=f \circ \tau .
$$

Given any function $f$ on TwoTall, we can symmetrize under $\tau$ to get a $\tau$ invariant function

$$
\sigma^{\operatorname{Tetra}}(f)=\frac{1}{4}(f+f \circ \tau+f \circ \tau \circ \tau+f \circ \tau \circ \tau \circ \tau),
$$


which we think of as a function on Tetra. Similarly, we can get functions on Didi via the symmetrization

$$
\sigma^{\operatorname{Didi}}(f)=\frac{1}{4}\left(f+f \circ \rho_{x}+f \circ \rho_{y}+f \circ \rho_{z}\right) .
$$

Now any function $f$ on TwoTall can be written as a Fourier series:

$$
f(x, y, z)=\sum_{(a, b, c) \in \mathbf{Z} \times \mathbf{Z} \times \frac{1}{2} \mathbf{Z}} \hat{f}(a, b, c) \exp (2 \pi i(a x+b y+c z)) .
$$

Note that the sum runs over the lattice $\mathbf{Z} \times \mathbf{Z} \times \frac{1}{2} \mathbf{Z}$, which is 'dual' to the original lattice $\mathbf{Z} \times \mathbf{Z} \times 2 \mathbf{Z}$ : The frequencies $a$ and $b$ in the $x$ and $y$ directions are integers, but the frequency $c$ in the $z$ direction is allowed to be a halfinteger, because the scale of the lattice in that direction is twice the scale in the $x$ and $y$ directions.

The Fourier basis functions $\phi_{a, b, c}=\exp (2 \pi i(a x+b y+c z)),(a, b, c) \in \mathbf{Z} \times \mathbf{Z} \times \frac{1}{2} \mathbf{Z}$ are eigenfunctions of the (positive) Laplacian $\Delta=-\left(\frac{\partial^{2}}{\partial x^{2}}+\frac{\partial^{2}}{\partial y^{2}}+\frac{\partial^{2}}{\partial z^{2}}\right)$ :

$$
\Delta \phi_{a, b, c}=4 \pi^{2}\left(a^{2}+b^{2}+c^{2}\right) \phi_{a, b, c} .
$$

Symmetrizing these Fourier basis functions under $\tau$ yields $\sigma^{\text {Tetra }}\left(\phi_{a, b, c}\right)$, a spanning set for the functions on Tetra. This spanning set is far from being a basis. For one thing, symmetrization lumps the basis functions together in groups, generally of size four. More important, symmetrizing a basis function can kill it off altogether. For example,

$$
\sigma^{\text {Tetra }}\left(\phi_{0,0,1 / 2}\right)=\sigma^{\text {Tetra }}\left(\phi_{0,0,1}\right)=\sigma^{\text {Tetra }}\left(\phi_{0,0,3 / 2}\right)=0 .
$$

However, by eliminating such redundancies, we can prune down to a basis of (unnormalized) eigenfunctions $\sigma^{\text {Tetra }}\left(\phi_{a_{i}, b_{i}, c_{i}}\right)$ on Tetra, with corresponding eigenvalues $4 \pi^{2}\left(a_{i}^{2}+b_{i}^{2}+c_{i}^{2}\right)$.

Similarly, we can get a basis of eigenfunctions $\sigma^{\operatorname{Didi}}\left(\phi_{a_{i}^{\prime}, b_{i}^{\prime}, c_{i}^{\prime}}\right)$ on Didi, with corresponding eigenvalues $4 \pi^{2}\left(a_{i}^{\prime 2}+b_{i}^{\prime 2}+{c^{\prime}}_{i}^{2}\right)$. If we can arrange that

$$
a_{i}^{2}+b_{i}^{2}+c_{i}^{2}=a_{i}^{\prime 2}+b_{i}^{\prime 2}+c_{i}^{\prime 2}
$$

for all $i$, then we will have verified that Tetra and Didi are spectral twins.

To find such a correspondence, we will take advantage of the fact that our two symmetrization mappings lump the basis functions together in two different but very nearly compatible ways. This leads to a correspondence between eigenfunctions which is for the most part very straight-forward. There are only two exceptional cases that must be treated carefully. 
Let

$$
V_{a, b, c}=\left\langle\phi_{ \pm a, \pm b, \pm c}, \phi_{ \pm b, \pm a, \pm c}\right\rangle,
$$

where we're using angle brackets to denote linear span. Please observe that $V_{a, b, c}=V_{b, a, c}, V_{a, b, c}=V_{-a, b, c}$, etc. In the generic case, namely when $a, b, c \neq$ 0 and $|a| \neq|b|$, the vector space $V_{a, b, c}$ is 16-dimensional, and both $\sigma^{\text {Tetra }}$ and $\sigma^{\text {Didi }}$ lump the 16 basis functions together in groups of 4 . In this case $\sigma^{\text {Tetra }}\left(V_{a, b, c}\right)$ and $\sigma^{\text {Didi }}\left(V_{a, b, c}\right)$ are both 4-dimensional, and we can clearly take bases of these spaces and match them up.

If it were true that $\operatorname{dim} \sigma^{\text {Tetra }}\left(V_{a, b, c}\right)=\operatorname{dim} \sigma^{\operatorname{Didi}}\left(V_{a, b, c}\right)$, for all $(a, b, c) \in \mathbf{Z} \times$ $\mathbf{Z} \times \frac{1}{2} \mathbf{Z}$, we would be all set. In fact this equality holds as long as no two of the parameters $a, b, c$ vanish, because in these cases $\sigma^{\text {Tetra }}$ and $\sigma^{\text {Didi }}$ continue to lump the basis functions together in groups of 4 . Of course $\operatorname{dim} \sigma^{\text {Tetra }}\left(V_{0,0,0}\right)=$ $\operatorname{dim} \sigma^{\operatorname{Didi}}\left(V_{0,0,0}\right)=1$, so that case is no problem. And if $c$ is a half integer, then $\operatorname{dim} \sigma^{\operatorname{Tetra}}\left(V_{0,0, c}\right)=\operatorname{dim} \sigma^{\operatorname{Didi}}\left(V_{0,0, c}\right)=0$.

So the question comes down to how to handle the cases $V_{n, 0,0}$ and $V_{0,0, n}$, with $n$ a non-zero integer, which we may assume is positive. (Remember that negating any of $a, b, c$ does not change the space $V_{a, b, c}$. ) To extend the correspondence between eigenfunctions, we must take these remaining exceptional cases in combination. Here is how it goes.

Odd exceptional case When $n$ is a positive odd integer,

$$
\begin{aligned}
\sigma^{\text {Tetra }}\left(V_{n, 0,0}\right) & =\langle\cos 2 \pi n x+\cos 2 \pi n y\rangle ; \\
\sigma^{\text {Tetra }}\left(V_{0,0, n}\right) & =0 ; \\
\sigma^{\text {Didi }}\left(V_{n, 0,0}\right) & =0 \\
\sigma^{\operatorname{Didi}}\left(V_{0,0, n}\right) & =\langle\cos 2 \pi n z\rangle .
\end{aligned}
$$

Taken together, these cases contribute a single eigenfunction of eigenvalue $4 \pi^{2}$. $n^{2}$ to the spectra of both Tetra and Didi.

Even exceptional case When $n$ is a positive even integer,

$$
\begin{aligned}
\sigma^{\operatorname{Tetra}}\left(V_{n, 0,0}\right) & =\langle\cos 2 \pi n x+\cos 2 \pi n y\rangle ; \\
\sigma^{\operatorname{Tetra}}\left(V_{0,0, n}\right) & =\langle\exp (2 \pi i n z), \exp (-2 \pi i n z)\rangle ; \\
\sigma^{\operatorname{Didi}}\left(V_{n, 0,0}\right) & =\langle\cos 2 \pi n x, \cos 2 \pi n y\rangle ; \\
\sigma^{\operatorname{Didi}}\left(V_{0,0, n}\right) & =\langle\cos 2 \pi n z\rangle .
\end{aligned}
$$

Taken together, these cases contribute three independent eigenfunctions of eigenvalue $4 \pi^{2} \cdot n^{2}$ to both spectra. 
By matching up these exceptional cases as indicated, we finish the job of matching up eigenfunctions of Tetra and Didi, and thus concretely demonstrate that these spaces are spectral twins.

Note that our scheme for matching eigenfunctions involves some arbitrary, symmetry-breaking choices. This shows up clearly in the even exceptional case above, but it is an issue even in the 'generic' case. We will see this same kind of symmetry-breaking again when we look at the proof of isospectrality by way of the Selberg trace formula.

\section{Unique}

Tetra and Didi are, up to scale, the only pair of non-isometric isospectral platycosms: They are the two-and-only cosmic spectral twins. The proof, due to Rossetti, involves a case-by-case analysis of all possible spectral coincidences among and between platycosms of the 10 possible types. In [12, Rossetti and Conway give a streamlined version of this proof, using Conway's theory of lattice conorms as an organizing principle. As you would expect, the techniques used in proving uniqueness yield another proof that Tetra and Didi are spectral twins.

A key ingredient in the uniqueness proof is Schiemann's theorem 13 that there are no spectral twins among torocosms: If $\mathbf{R}^{3} / \Lambda_{1}$ and $\mathbf{R}^{3} / \Lambda_{2}$ are isospectral, then they (and the lattices $\Lambda_{1}$ and $\Lambda_{2}$ ) are isometric. Milnor's original example of spectral twins was a pair of 16-dimensional tori [10. Subsequently, lowerdimensional pairs of isospectral tori were found, culminating with the discovery of a 4-dimensional pair by Schiemann, simplified and extended to a 4-parameter family of pairs by Conway and Sloane [3. Schiemann showed that as far as tori are concerned, dimension 4 is the end of the line. By opening the field up to other flat manifolds, we can get down to dimension 3-but just barely!

\section{Selberg}

Here, as promised above, we outline a proof of isospectrality by way of the Selberg trace formula. The version of the trace formula that we want to use expresses the Laplace transform of the spectrum (or properly speaking, of the spectral measure) as the sum of contributions attributable to families of closed geodesics. To show that Tetra and Didi are isospectral, we will examine the closed geodesics of each, and check that the total spectral contribution of Tetra's geodesics is just the same as that of Didi's. 


\begin{tabular}{|c|c|c|c|c|c|c|c|c|c|c|c|c|c|c|c|c|c|}
\hline \multirow[b]{2}{*}{$l$} & \multicolumn{10}{|c|}{ Tetra } & \multicolumn{7}{|c|}{ Didi } \\
\hline & $w_{l}$ & $n$ & $t$ & $k$ & $w$ & $n$ & $t$ & $k$ & $w$ & $n$ & $t$ & $k$ & $w$ & $n$ & $t$ & $k$ & $w$ \\
\hline$\frac{1}{2}$ & 4 & 2 & $\frac{1}{4}$ & 1 & $2 \cdot \frac{2}{1}$ & & & & & 4 & $\frac{1}{2}$ & 1 & $4 \cdot \frac{1}{1}$ & & & & \\
\hline 1 & 2 & 2 & $\frac{1}{2}$ & 2 & $2 \cdot \frac{1}{2}$ & 1 & $\frac{1}{2}$ & 1 & $1 \cdot \frac{1}{1}$ & & & & & 2 & $\frac{1}{2}$ & 1 & $2 \cdot \frac{1}{1}$ \\
\hline$\frac{3}{2}$ & $\frac{4}{3}$ & 2 & $\frac{1}{4}$ & 3 & $2 \cdot \frac{2}{3}$ & & & & & 4 & $\frac{1}{2}$ & 3 & $4 \cdot \frac{1}{3}$ & & & & \\
\hline 2 & 0 & & & & & & & & & & & & & & & & \\
\hline$\frac{5}{2}$ & $\frac{4}{5}$ & 2 & $\frac{1}{4}$ & 5 & $2 \cdot \frac{2}{5}$ & & & & & 4 & $\frac{1}{2}$ & 5 & $4 \cdot \frac{1}{5}$ & & & & \\
\hline 3 & $\frac{2}{3}$ & 2 & $\frac{1}{2}$ & 6 & $2 \cdot \frac{1}{6}$ & 1 & $\frac{1}{2}$ & 3 & $1 \cdot \frac{1}{3}$ & & & & & 2 & $\frac{1}{2}$ & 3 & $2 \cdot \frac{1}{3}$ \\
\hline$\frac{7}{2}$ & $\frac{4}{7}$ & 2 & $\frac{1}{4}$ & 7 & $2 \cdot \frac{2}{7}$ & & & & & 4 & $\frac{1}{2}$ & 7 & $4 \cdot \frac{1}{7}$ & & & & \\
\hline 4 & 0 & & & & & & & & & & & & & & & & \\
\hline$\frac{9}{2}$ & $\frac{4}{9}$ & 2 & $\frac{1}{4}$ & 9 & $2 \cdot \frac{2}{9}$ & & & & & 4 & $\frac{1}{2}$ & 9 & $4 \cdot \frac{1}{9}$ & & & & \\
\hline
\end{tabular}

Table 1: Balancing geodesics. This table shows the balancing of the spectral contributions from the twisted geodesics in Tetra and Didi. Here $l$ is length, and $w_{l}$ the total spectral contribution (weight) of geodesics of length $l$, measured in units of the spectral contribution of a primitive half-twisting geodesic of length $l$. The point of this table is to demonstrate that $w_{l}$ is the same for Tetra and Didi. For geodesics of a specific kind, $n$ tells the number of geodesics; $t$ the twist (either $\frac{1}{4}$ or $\frac{1}{2}$ ); $k$ the imprimitivity exponent; and $w$ the aggregate spectral weight for geodesics of this kind. An individual geodesic with imprimitivity exponent $k$ has weight $1 / k$ if it is half-twisting, and $2 / k$ if it is quarter-twisting. Weights do not depend on the handedness of the twist, so we do not distinguish between 1/4-twisting and 3/4-twisting geodesics.

The relevant computations are indicated in Table 1. Here we will explain informally what lies behind the computations in the table. The discussion is contrived in such a way as to allow us to put off actually writing down Selberg's formula until after we have put it to use. Our reason for preferring this inverted approach is that (in the present case, at least) the Selberg formula is easier to apply than to state.

Recall that when it comes to the shortest geodesics, which have length $1 / 2$, Tetra has two 'quarter-twisters', while Didi has four 'half-twisters'. Now it happens that, in a flat 3-manifold, the spectral contribution of any primitive quarter-twisting geodesic is just twice that of a primitive half-twisting geodesic. (This is an aspect of a general phenomenon: 'The more the twist; the less the contribution.') So as far as the shortest geodesics go, the contributions to the 
spectrum are the same.

Next come geodesics of length 1 . Both Tetra and Didi have two 2-dimensional families of non-twisting geodesics, which they inherit from the common cover TwoTall. These common families of non-twisting geodesics make identical contributions to the spectrum. In general, the non-twisting geodesics in Tetra and Didi are all inherited from the common cover, and consequently contribute equally to their spectra. So we don't have to worry about non-twisting geodesics.

Looking at twisted geodesics of length 1 , the only kind that arise are halftwisters. In Tetra we already identified one primitive half-twister running vertically up the midlines of the sides of the box we have chosen as our fundamental domain; in Didi, we have two primitive half-twisters sitting in the vertical Klein bottle. That's it, as far as primitive geodesics are concerned. However, in Tetra, we also have two imprimitive half-twisters, gotten by running twice around those two primitive quarter-twisters of length $1 / 2$. In the Selberg formula the spectral contribution of an imprimitive geodesic must be divided by its degree of imprimitivity or exponent, which is the number of times it runs around its primitive ancestor. So the spectral contribution of Tetra's one new half-twister and two recycled quarter-twisters just matches that of Didi's two brand new half-twisters.

Next among twisted geodesics are those of length $3 / 2$. Here we are back to balancing Tetra's two quarter-twisters, now thrice-imprimitive, against Didi's four half-twisters, also thrice-imprimitive.

At length 2, there are no non-twisting geodesics.

Length $5 / 2$ is like $1 / 2$ and 3/2: Tetra has two quarter-twisters and Didi four half-twisters, all now five-times-imprimitive

Length 3 is like length 1: We are back to balancing Didi's two half-twisters, now thrice-imprimitive, against Tetra's one half-twister, now thrice-imprimitive, and two quarter-twisters, now recycled as six-times-imprimitive half-twisters.

And so it goes on up the line. Thus Tetra and Didi are isospectral.

\section{Formula}

We have chosen to describe the geodesic balancing act between Tetra and Didi in words, rather than symbols, in order to put off having to state explicitly Selberg's formula for platycosms. The goal was to show how you can use the 
formula without having to know precisely what the formula is. Now, here comes the formula.

Let $M=\mathbf{R}^{3} / \Gamma$ be a platycosm with covering group $\Gamma$, and let $\Lambda \subset \Gamma$ be the lattice subgroup of $\Gamma$. Let $0=\lambda_{0}<\lambda_{1} \leq \lambda_{2} \leq \lambda_{3} \leq \ldots$ be the sequence of eigenvalues of the Laplacian on $M$, where as usual multiple eigenvalues are listed multiple times in the sequence. For a geodesic $g$, let $l(g)$ be its length, $\theta(g)$ its twist (in radians), and $k(g)$ its imprimitivity exponent. Let $G$ be the set of (nontrivially) twisted closed geodesics of $M$. Here again, we take no notice of orientation: oppositely-oriented geodesics are considered to be the same.

The version of the Selberg trace formula we need relates the spectrum of $M$ to the geometry of $M$, by giving two separate ways to compute a certain function $K(t)$, the 'trace of the heat kernel'. The first way is in terms of the spectrum, while the second way is in terms of the geometry. For present purposes, we don't need to know just what $K(t)$ is - just that the two expressions are equal.

Here's the formula:

$$
K(t)=\sum_{n} e^{-\lambda_{n} t}=\int_{0}^{\infty} \frac{1}{(4 \pi t)^{\frac{3}{2}}} e^{-\frac{s^{2}}{4 t}} \mathrm{~d} N(s)
$$

where

$$
N(s)=\operatorname{Vol}(M)|\{\gamma \in \Lambda:|\gamma| \leq s\}|+2 \sum_{g \in G} \frac{1}{k(g)} V_{l(g), \theta(g)}(s)
$$

where

$$
V_{h, \theta}(s)=\left\{\begin{array}{ll}
0, & 0 \leq s<h \\
h \pi \frac{s^{2}-h^{2}}{\left(2 \sin \frac{\theta}{2}\right)^{2}}, & h \leq s
\end{array} .\right.
$$

Here $V_{h, \theta}(s)$ is the volume of a cylinder of height $h$ and $\theta$-twisted height $s$, which we define as follows: If the sides of a cylinder of height $h$ are replaced with parallel segments, and the top is twisted through an angle $\theta$ relative to the bottom, the segments stretch to form (part of) a hyperboloid of one sheet; their stretched length is what we're calling the $\theta$-twisted height. (See Figure 3.)

Note the factor of 2 in front of the sum over $G$ in the formula for $N(s)$. This arises because of our lumping together oppositely-oriented geodesics. When it comes to doing explicit computations using this formula, this lumping appears unnatural, because translating one way around a geodesic is not at all the same thing as translating the other way. But for us to try to enforce a distinction between oppositely-oriented geodesics would only cause confusion, because it 
would run counter to established practice. Nobody is going to be comfortable with the notion that Tetra has four geodesics of length $\frac{1}{2}$.

Now, what makes Selberg's formula so useful is the fact that the function $K(t)=$ $\sum_{n} e^{-\lambda_{n} t}$ determines the spectrum: In fact, it is the Laplace transform of a mass distribution with a unit mass placed at every eigenvalue, so we can recover the spectrum by inverting a Laplace transform. The trace formula thus shows that the Laplace spectrum is determined by the function $N(s)$, which is computed directly from geometrical data.

The use we made above of the trace formula depended essentially on only two of its properties. First is the amazing fact that the contribution of a twisted geodesic to $N(s)$ depends on the twist $\theta$ only through the factor $\frac{1}{\sin ^{2} \frac{\theta}{2}}$. For a half-twisting geodesic $(\theta=\pi)$, this factor is 1 ; for a quarter-twisting geodesic $(\theta=\pi / 2)$, this factor is 2 . Second is the way recycled geodesics get only partial credit in $N(s)$, because of the factor $\frac{1}{k(g)}$.

Please observe that, since we are only using this formula to show that the two spaces Tetra and Didi are isospectral, it isn't crucial that we have the formula exactly right. If the formula as written were off by a constant factor here or there, say a stray factor of 2 or $\sqrt{\pi}$, it would do us no harm, as long as the error applied equally to Tetra and Didi. That's good, of course. Working only with 'higher-level' properties of the formula means that we're approaching things in a 'conceptual' way, and insulating ourselves from possible bugs in the formula. The drawback is that we haven't given the formula a real workout.

Exercise Give the formula a real workout by using it to compute explicitly the heat trace for TwoTall, and then Tetra. (No need to check Didi, since we've already checked that the answer will be the same for Didi as for Tetra.) Observe that

$$
K_{\text {Tetra }}-\frac{1}{4} K_{\text {TwoTall }}=K_{\mathbf{R} / \frac{1}{2} \mathbf{Z}}-\frac{1}{4} K_{\mathbf{R} / 2 \mathbf{Z}} \text {. }
$$

Explain this remarkable 'effective 1-dimensionality' of the relationship between Tetra and TwoTall.

\section{Derivation}

So that's the Selberg trace formula for platycosms, or rather, one particular form of it. As for the derivation, equivalent formulas are derived in the papers of Gangolli [4] and Bérard-Bergery [1]. The formula can also be derived by adapting the classical Poisson summation formula, which was the original 
inspiration for the Selberg formula: This is the approach of Sunada [14 and Miatello and Rossetti [9].

More mundanely, the formula can also be written down by identifying the Laplace transform of the spectrum with the trace of the heat kernel in the usual Selbergian way, and evaluating the diagonal of the heat kernel using the 'method of images' from sophomore physics. Pairs of images lying within a given distance of each other fall into cylinders of given 'twisted height'. Measuring the volume of these cylinders requires only the Pythagorean theorem and the usual formula for the volume of a cylinder. The fact that a quarter-twisting geodesic contributes twice as much to the spectrum as a half-twisting geodesic boils down to the fact that a cylinder of given height $h$ and quarter-twisted height $s$ has twice the volume of a cylinder with the same $h$ and half-twisted height $s$. (See Figure 3.)
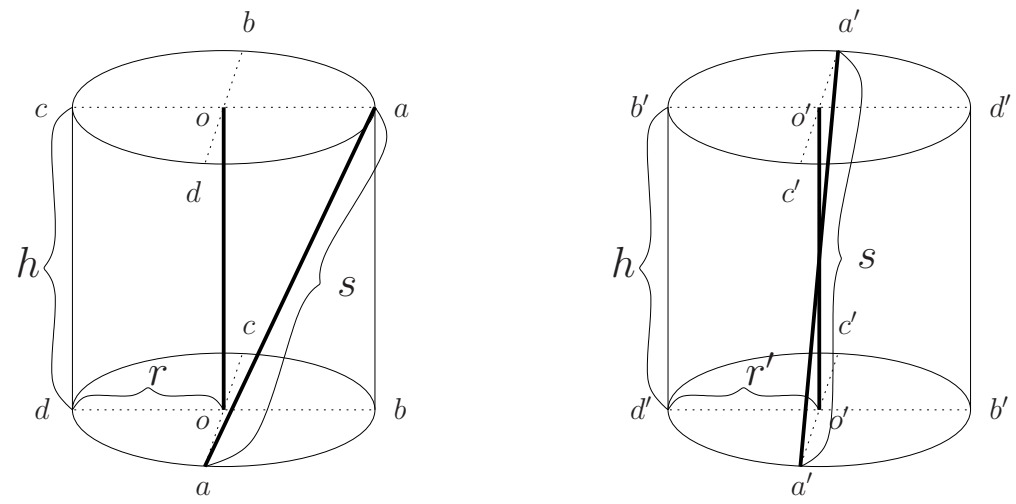

Figure 3: Volume of cylinders with given height $h$ and twisted height $s . \operatorname{Vol}_{\frac{1}{4}}=\pi r^{2} h$; $\operatorname{Vol}_{\frac{1}{2}}=\pi r^{\prime 2} h$. By the Pythagorean theorem, $h^{2}+2 r^{2}=s^{2}$ and $h^{2}+4 r^{\prime 2}=s^{2}$. So $\mathrm{Vol}_{\frac{1}{4}}^{2}=2 \mathrm{Vol}_{\frac{1}{2}}$.

A similar hands-on approach to Selberg's formula works for hyperbolic 2- and 3-manifolds (or orbifolds), with the hyperbolic law of cosines taking the place of the Pythagorean theorem.

\section{Subtle isospectrality}

The remarkable Selbergian interplay between the geodesics in Tetra and Didi is what we were looking for when we discovered this pair. Originally, we were interested in finding (or ruling out) an analogous pair of hyperbolic 3manifolds. There are plenty of examples of spectral twins among hyperbolic 
3-manifolds, but the standard methods for producing spectral twins yield pairs whose geodesics have matching lengths and twists. Selberg's formula seems to allow the possibility of twins that are subtly isospectral, meaning, 'isospectral, but not merely by virtue of having geodesics with matching lengths and twists'.

After some fruitless attempts to find such a pair among hyperbolic 3-manifolds, we tried looking among flat 3-manifolds instead.

Now, the precise definition of 'subtle isospectrality' for flat manifolds is a subtle business, because various of the geodesics in flat manifolds come in parallel families, and you have to be careful to choose the right definition for the 'multiplicity' of such geodesics, which you will need in order to decide whether a given pair of manifolds have geodesics with 'matching' lengths and twists. Of course, you could make any definition you want, and then investigate it. But to be interesting, the definition of multiplicity should end up meaning that two manifolds are subtly isospectral just if they are isospectral, but not merely because of some straight-forward way of matching contributions to Selberg's formula.

We don't need to fuss about this here, because however you decide to measure multiplicity, Tetra and Didi will have different multiplicities of geodesics of the shortest length. That's because these shortest geodesics are all isolated, on account of their twisting. Thus Tetra and Didi are subtly isospectral - and no other pairs of non-isometric platycosms are isospectral, whether subtly or not.

The possibility of subtly isospectral hyperbolic 3-manifolds remains open.

\section{Remarks}

Misconception about Selberg for hyperbolic 3-manifolds Some people mistakenly believe that, for a hyperbolic 3-manifold, Selberg's formula allows you to read off from the spectrum the lengths and twists of the closed geodesics. For example, Reid [1] cites Gangolli 4] and Bérard-Bergery [1] in support of this assertion. Neither Gangolli nor Bérard-Bergery makes such a statement, and Gangolli in particular is explicit about the fact that the Selberg formula leaves open the possibility of an example of the kind we were (and still are) looking for.

This misconception most likely stems from conflicting uses of the term length spectrum, which we have been studiously avoiding here. 
Hyperbolic surfaces In another attempt to work up to hyperbolic 3-manifolds, we looked at hyperbolic surfaces. We were disappointed to find that no subtly isospectral pairs exist among hyperbolic surfaces. The question only becomes interesting in the case of non-orientable surfaces, because the Selberg formula immediately implies that for hyperbolic surfaces, spectral twins always have matching lengths. For non-orientable surfaces, it is possible to construct a plausible scenario for matching the contributions of the geodesics of a pair of surfaces in a way similar to that of Tetra and Didi, where geodesics don't have matching lengths. But in the end it proves impossible to make this work: We show that to balance the spectral contributions of geodesics on a pair of surfaces whose geodesics can't be matched so as to preserve length and orientability class, you would need the number of geodesics with length at most $l$ to be asymptotically at least $2 e^{l} / l$; but by results of Huber [6, 7] and others, this number must be asymptotically exactly $e^{l} / l$, which is a factor of 2 short of what we need. (Huber's results are stated only for orientable surfaces, but they hold as well in the non-orientable case.)

Thus, while it seems like we come tantalizingly close to being able to build a pair of hyperbolic surfaces that are subtly isospectral, in the end we are forced to conclude that any pair of spectral twins among hyperbolic surfaces must have geodesics with matching lengths and twists.

Laplacian on forms While Tetra and Didi are isospectral for the usual Laplacian acting on functions, they are not isospectral for the Laplacian acting on 1 -forms or 2 -forms. This is a simple consequence of the techniques of Miatello and Rossetti (see Theorem 3.1 of [8]). However, this can also be seen immediately because the Betti number $b_{k}$ equals the multiplicity of the eigenvalue 0 of the Laplacian acting on $k$-forms. Tetra and Didi have distinct $b_{1}$, and hence distinct spectrum on 1 -forms. Since they are both orientable 3 -manifolds, they have $b_{1}=b_{2}$, so they have distinct $b_{2}$ and distinct spectrum on 2-forms. Examples of manifolds which are isospectral but have distinct first Betti numbers, or which are isospectral on functions but not on 1-forms, were previously known only in higher dimensions (see [5] and the references therein).

Acknowledgement The second author is supported by Conicet and a Guggenheim fellowship. 


\section{References}

[1] Lionel Bérard-Bergery, Laplacien et géodésiques fermées sur les formes d'espace hyperbolique compactes, from: "Séminaire Bourbaki, 24ème année (1971/1972), Exp. No. 406", Lecture Notes in Math. 317, Springer-Verlag (1973) 107-122 MathReview

[2] John Horton Conway, Juan Pablo Rossetti, Describing the platycosms, arXiv:math.DG/0311476

[3] John Horton Conway, Neil J A Sloane, Four-dimensional lattices with the same theta series, Internat. Math. Res. Notices 4 (1992) 93-96 MathReview

[4] Ramesh Gangolli, The length spectra of some compact manifolds of negative curvature, J. Differential Geom. 12 (1977) 403-424 MathReview

[5] Carolyn S Gordon, Isospectral manifolds with different local geometry, J. Korean Math. Soc. 38 (2001) 955-970 MathReview

[6] Heinz Huber, Zur analytischen Theorie hyperbolischer Raumformen und Bewegungsgruppen, I, Math. Ann. 138 (1959) 1-26 MathReview

[7] Heinz Huber, Zur analytischen Theorie hyperbolischer Raumformen und Bewegungsgruppen, II, Math. Ann. 143 (1961) 463-464 MathReview

[8] Roberto J Miatello, Juan Pablo Rossetti, Flat manifolds isospectral on p-forms, J. Geom. Analysis 11 (2001) 647-665 MathReview

[9] Roberto J Miatello, Juan Pablo Rossetti, Length spectra and p-spectra of compact flat manifolds, J. Geom. Analysis 13 (2003) 631-657 MathReview

[10] John Milnor, Eigenvalues of the Laplace operator on certain manifolds, Proc. Nat. Acad. Sci. USA 51 (1964) 542 MathReview

[11] Alan W Reid, Isospectrality and commensurability of arithmetic hyperbolic 2 and 3 -manifolds, Duke Math. J. 65 (1992) 215-228 MathReview

[12] Juan Pablo Rossetti, John Horton Conway, Hearing the platycosms, arXiv:math.DG/0311470

[13] Alexander Schiemann, Ternary positive definite quadratic forms are determined by their theta series, Math. Ann. 308 (1997) 507-517 MathReview

[14] Toshikazu Sunada, Spectrum of a compact flat manifold, Comment. Math. Helv 53 (1978) 613-621 MathReview

[15] Jeffrey R Weeks, The Shape of Space, Second Edition, Monographs and Textbooks in Pure and Applied Mathematics 249, Marcel Dekker, New York (2002) MathReview

[16] Jeffrey $\mathbf{R}$ Weeks, Real-time rendering in curved spaces, IEEE Computer Graphics and Applications 22 (Nov/Dec 2002) 90-99 